\title{
Prevention and treatment of febrile neutropenia
}

\author{
F. Weissinger ${ }^{1}$, W. J. Heinz ${ }^{2}$ \\ 1Department of Hematology/Oncology and Pallative Medicine, Center of Internal Medicine, Evangelisches Krankenhaus \\ Bielefeld, Bielefeld, Germany \\ 2Department of Internal Medicine II, University of Würzburg, Würzburg, Germany
}

Received 6 November 2011; accepted 5 March 2012

Febrile Neutropenia is a relevant factor for mortality after chemotherapy. Dependent upon factors like duration of neutropenia ( $\geq 7 \mathrm{~d}$ means high-risk situation), patient- and disease specific criteria e.g. defined in the MASCC score (Multinational Association of Supportive Care in Cancer) the individual risk for the patient for the need of being hospitalized can be estimated. Antimicrobial therapy has to be started immediately, to avoid high mortality. Empiric or calculated antiinfective therapy includes broadspectrum-lactam antibiotics in first-line. After 72 to $96 \mathrm{~h}$ of treatment, when the patients are still febrile and clinically not improving, an empiric switch of the antibiotic treatment can be considered, e.g. closing the gaps in the gram-positive spectrum. In this situation high risk patients also should receive an antifungal agent with activity against aspergillus spp. Strategies to prevent infection in neutropenia are antiinfective prophylaxis or the use of G-CSF (granulocyte-colony stimulating factor). Antibiotic prophylaxis might help to prevent infections and to improve outcome esp. for high risk patients. Antifungal prophylaxis including Aspergillus species is recommended in the situation of induction therapy for acute myelogenic leukemia. Patients, expecting a phase of prolonged neutropenia with a history of mold infection should receive a secondary antifungal prophylaxis.

Keywords: Febrile neutropenia, emprical antiinfective treatment, antiinfective prophylaxis, granulocyte-colony stimulating factor.

\section{Introduction}

Neutropenia after chemotherapy is one of several important risk factors for infection. The infection risk depends upon the duration of neutropenia and other patient-specific or therapy-specific factors. The most common symptom of infection is fever. Documented evidence of infection is present in only about $40 \%$ of patients $[1,2]$. Physicians are often confronted with fever of unknown origin, and the therapeutic regimens are empiric or calculated on the basis of therapeutic trials. Because of the risk of rapid deterioration, empiric anti-infec-

Correspondence: Florian Weissinger, MD, Evangelisches Krankenhaus Bielefeld, Schildescher Str. 99, 33611 Bielefeld, Germany. E-mail: Florian.Weissinger@evkb.de tive therapy must be started immediately. Here we discuss recommendations for the diagnosis, therapy and prophylaxis of febrile neutropenia after chemotherapy, excluding the context after allogenic stem-cell transplantation.

\section{Febrile neutropenia}

\section{Definitions - risk assessment}

Neutropenia is classified in intrinsic defects, like cyclic neutropenia, and acquired causes. In this article, we focus upon chemotherapy-induced neutropenia, the major cause of acquired neutropenia. Clinically significant neutropenia, associated with an increased risk for infection, is defined as a neutrophil count below $500 / \mu \mathrm{l}$, whereas a count below $100 / \mu \mathrm{l}$ is considered as severe neutropenia. Patients with an absolute neutrophil count predicted to drop to below $500 / \mu \mathrm{l}$ in the next two days are also considered as having significant neutropenia. In terms of chemotherapy-induced neutropenia, the discrimination between a duration of less than or equal 7 days (referred to as a standard risk for infection) and a prolonged duration of over 7 days (referred to as a high risk for infection, i.e. after induction therapy for acute myelogenic leukaemia) [3] is relevant to the management of neutropenia. Patients after autologous stem cell transplantation cannot be explicitly assigned to either of these groups, because the duration of neutropenia is usually between 6 and 10 days. We shall not address the unique situation after allogenic transplantation in this report.

Inclusion of additional parameters predicting the outcome of febrile neutropenia led to a scoring system (Multinational Association for Supportive Care in Cancer = MASCC Score) that may define a carefully selected patient group for outpatient antibiotic treatment (Table 1; a risk score of $>2.1$ identifies low risk patients) $[4,5]$.

Fever in neutropenia is defined as an oral temperature of $38.3^{\circ} \mathrm{C}$ once, or a temperature of $38.0^{\circ} \mathrm{C}$ twice within $12 \mathrm{~h}$ or lasting longer than $1 \mathrm{~h}$. Even if there are other possible reasons for fever-like B-symptoms, reaction against medication or blood products, it must be assumed that the cause of fever in neutropenia is an infection. Fever occurs in 10 to $50 \%$ of patients after chemotherapy for solid tumours during neutropenia and in more than $\mathbf{8 0 \%}$ after chemotherapy for haematological malignancies [6]. Some patients do not develop 


\begin{tabular}{|c|c|}
\hline Characteristic & Weight \\
\hline Burden of febrile neutropenia with no or mild symptoms* & 5 \\
\hline No hypotension (systolic blood pressure $>90 \mathrm{mmHg}$ ) & 5 \\
\hline No chronic obstructive pulmonary disease ${ }^{\dagger}$ & 4 \\
\hline $\begin{array}{l}\text { Solid tumour or haematological malignancy with no previous } \\
\text { fungal infection } \ddagger\end{array}$ & 4 \\
\hline No dehydration requiring parenteral fluids & 3 \\
\hline Burden of febrile neutropenia with moderate symptoms* & 3 \\
\hline Outpatient status & 3 \\
\hline Age $<60$ years & 2 \\
\hline \multirow{2}{*}{\multicolumn{2}{|c|}{$\begin{array}{l}\text { NOTE. The maximum value of the score is } 26 \text {. } \\
\text { *Burden of febrile neutropenia: Refers to the patient's general clinical } \\
\text { status as influenced by the febrile neutropenic episode. It should be } \\
\text { evaluated on the following scale: no or mild symptoms (score of 5); } \\
\text { moderate symptoms (score of 3); severe symptoms or moribund (score } \\
\text { of 0). Scores of } 3 \text { and } 5 \text { are not cumulative. } \\
\text { tChronic obstructive pulmonary disease: Means active chronic bronchitis, } \\
\text { emphysema, decrease in forced expiratory volumes, need for oxygen } \\
\text { therapy and/or steroids and/or bronchodilatators requiring treatment at } \\
\text { the presentation of the febrile neutropenic episode. } \\
\text { fPrevious fungal infection: Means demonstrated fungal infection or }\end{array}$}} \\
\hline & \\
\hline $\begin{array}{l}\text { ‡Previous fungal infection: Means demonstrated fungal infectio } \\
\text { empirically-treated suspected fungal infection. }\end{array}$ & \\
\hline
\end{tabular}

fever (i.e. under treatment with steroids or pain medication like novaminsulfon), but show signs of infections. These patients should be treated in the same way as with fever in neutropenia.

\section{Diagnostic evaluation}

In the presence of fever, in accordance with the aforementioned definition, immediate anti-infective treatment should be started and diagnostics be performed. Diagnostics should include clinical examination (mandatory every day or twice a day), assessing any changes in skin and mucosa, exit sites of central and peripheral venous access, upper and lower respiratory tracts, urogenital tract, abdomen and perianal region, and the monitoring of blood pressure, pulse rate and respiratory frequency.

- At least 2 pairs of blood cultures: two from each lumen of central venous access and at least two from a peripheral vein.

- Complete blood cell count including neutrophil count and platelets

- Serum levels of creatinine, electrolytes, ALAT, ASAT, alkaline phosphatase, gamma-GT, bilirubin, INR, PTT, CRP

- Other cultures from sites of suspected infections

- A chest CT scan (alternatively chest X-ray) in case of pulmonary symptoms

\section{Anti-infective treatment of febrile neutropenia}

Fever occurs in $10-50 \%$ of patients after chemotherapy for solid tumours during neutropenia and in more than $80 \%$ after chemotherapy for haematological malignancies. Despite diagnostic procedures, the source of infection can be identified in only $40 \%$ of fever episodes $[1,2]$ and only $23 \%$ of episodes are associated with a positive blood culture [7]. Therefore, the
Tab. 2: Most common pathogens in

neutropenic patients

Gram-negative pathogens

Escherichia coli (including ESBL pathogens)

Klebsiella species (including ESBL pathogens)

Enterobacter species

Pseudomonas aeruginosa

Citrobacter species

Acinetobacter species

Stenotrophomonas maltophilia

Gram-positive pathogens

Coagulase-negative staphylococci

Staphylococcus aureus (including MRSA)

Enterococcus species (including VRE)

Other Streptococci (S. viridans, S. pneumoniae, S. pyogenes)

Corynebacteria species

Less frequent are anaerobic pathogens

Clostridium species

Bacteroides species

Fusobacterium species

Propionibacterium species

MRSA methicillin resistent staphylococci; VRE vancomycin resistent

enterococci; $E S B L$ extended-spectrum $\beta$-lactamase.

spectrum of empiric antimicrobial treatment must cover gram-negative aerobic bacteria (enterobacteriaceae and pseudomonas aeruginosa), gram-positive cocci (streptococci, staphylococcus aureus and enterococci), and it should be adapted to the local resistance profile (i.e. MRSA, VRE and ESBL-producing bacteriae). Anaerobic pathogens or fungi do not have to be considered in the first-line empiric anti-infective regimen. The most common pathogens are listed in Table 2 [8]. Without antibiotic prophylaxis, gram-negative cocci are the most often isolated pathogens. When using antibiotic prophylaxis, there is a trend toward gram-positive cocci [9]. The interpretation of microbiological results must consider that positive cultures for coagulase-negative staphylococci or corynebacteriae often resemble contamination. It is therefore recommended that only two positive cultures detecting these pathogens be considered as relevant. Furthermore, results from positive cultures should be used to include these pathogens in the anti-infective regimen, but not to narrow the treatment's spectrum.

Anti-infective therapy must be initiated immediate, because any delay can worsen the prognosis of patients with infection in neutropenia [10]. It is important not to wait for diagnostic findings before starting therapy.

The ideal choice of empiric antibiotics is a broad-spectrum regimen, covering the most common pathogens. Single agent regimens have been proven to be equivalent to combination treatment [11-20]. Thus a first-line treatment with a pseudomonas aeroginosa-covering $\beta$-lactam antibiotic is recommended. Specifically, piperacillin/tacobactam, ceftazidime, cefepime, imipenem or meropenem are recommended. Because of the increase in ESBL-producing organisms, the use of cephalosporins is questionable [21], and there is reluctance to use cefepime, because that agent might be associated with an increase in mortality compared to alternative antibiotic regimens [22]. A combination with aminoglycosides is discouraged [23]. The addition of glycopeptides should be restricted to patients with suspected se- 
Fever $\left(\geq 38.3^{\circ} \mathrm{C}\right.$ or $\left.2 \times \geq 38.0^{\circ} \mathrm{C}\right)$ in Neutropenia $(<500$ neutrophils/ $/ \mathrm{ll})$

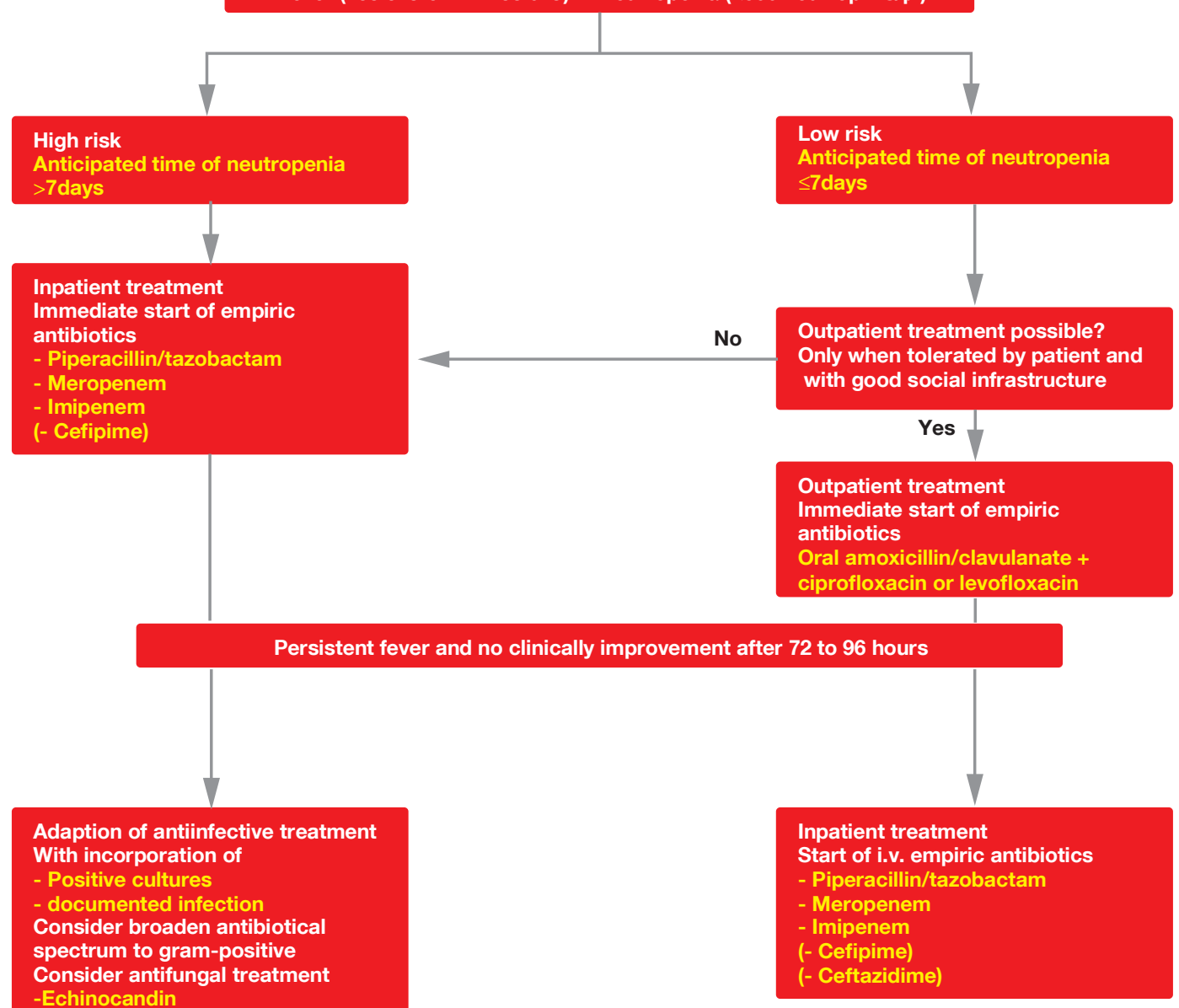

-Lip. Amphotericin B

-Voriconazole

Consider switch class of antifungal

treatment after prophylaxis

Fig. 1: Algorithm of treatment decision for febrile neutropenia

vere mucositis, skin or soft-tissue infection, or a suspected foreign body (e.g. central venous access)-related infection. Included in empirical first-line treatment, it does not improve outcome [24]. The median time to defervescence in patients with haematological malignancies is about 5 days [25], and in patients with solid tumours and low risk about 2 days [26].

For patients with persistent fever and no clinical improvement after 72 to 96 hours, it is recommended to repeat the aforementioned diagnostic procedures and to switch an empirical anti-infective regimen if there is no detectable infection source. The gaps in the gram-negative spectrum should be closed. Thus after piperacillin/tacobactam or cephalosporine as first-line, imipenem or meropenem is a reasonable choice. After first-line carbapenem, the addition of an aminoglycoside or glycopeptide should be considered. In the high-risk group (expected duration of neutropenia $>7$ days) the addition of empiric antimycotic treatment should be considered if the infection is not under control after 4 to 7 days of antibiotic treatment. A pre-emptive approach for high-risk patients using information from CT scans and blood tests (such as the serum galactomannan test in combination with clinical evidence) have resulted in reduced antifungal treatment ( $7.7 \%$ of patients) compared to an empirical strategy (35\% qualified for empirical antifungal treatment) without compromising patient outcomes [27]. Liposomal amphotericin-B, caspofungin or voriconazol are reasonable choices for empirical antifungal treatment in high-risk patients showing a risk for aspergillus infection. Clinically unstable patients on antimycotic prophylaxis active against aspergillus should switch to empirical treatment with an antifungal agent of another class. Unless there is a documented or suspected viral infection, no antiviral therapy is indicated.

Only those patients with an expected duration of neutropenia of under 7 days and assignment to the low-risk category according to the MASCC score should be managed in an outpatient setting with oral antibiotics (amoxicillin/clavulanate in combination with ciprofloxacin or levofloxacin) $[4,28]$. Fluorochinolones have gaps in the gram-positive spectrum and should not be used as single therapy. Also, there is no indication for fluorochinolones after fluorochinolone prophylaxis. If there is no defervescence and the patients are not clinically stable, they should be hospitalised and intravenous antibiotic treatment must be started empirically, e.g. with piperacillin/tazobactam. 
Anti-infective treatment must be continued for two days after defervescence and a neutrophil count $>500 / \mu$ l. Documented infections may be handled differently, as pneumonias or soft-tissue infections must be treated for 10 to 14 days, independently from neutrophil recovery.

We do not specifically address herein all situations involving documented infection, but the anti-infective regimen should cover the documented infection and still cover the broad spectrum of empiric treatment.

\section{Role of haematopoietic growth factors}

Concerning neutropenic fever, G-CSF (granulocyte-colonystimulating factor) is the most important haematopoietic growth factor worthy of discussion.

In several studies, the use of G-CSF has been shown to reduce the incidence of neutropenic fever and infection-related mortality. According to a meta-analysis of 17 randomised trials, most demonstrated a benefit of G-CSF independent of the basic risk of febrile neutropenia in the specific trial [29]. Thus it is recommended that G-CSF be used as primary prophylaxis in patients undergoing chemotherapy with a risk of $\geq 20 \%$ febrile neutropenia [3], particularly in those with additional risk factors like older age, comorbidities such as liver, kidney, lung or cardiac disease, poor Karnofsky performance status, or an impaired immune system. After an episode of febrile neutropenia, patients should receive G-CSF after the next course of chemotherapy as secondary prophylaxis. There is no indication for prophylactic G-CSF with a risk of febrile neutropenia of $\leq 10 \%$. Patients in the risk group between 10 and $20 \%$ should be evaluated individually regarding the other risk factors.

In the specific situation after autologous stem cell transplantation, where patients have a risk of neutropenic fever of approximately $60 \%$, G-CSF reduces the risk of fever, infection, reduces the number of days on antibiotic treatment, but has no significant influence on outcome and mortality. Its use is therefore optional.

The interventional approach starting G-CSF in the context of neutropenic fever has not been shown to improve outcome. The results for reducing the days on antibiotics and duration of hospitalisation are inconsistent [30, 31]. None of the guidelines (2010 update of the Guideline by the "Infectious Diseases Society of America" (IDSA) and Guideline of the "Deutsche Gesellschaft fuer Haematologie und Onkologie" (DGHO)) advocate using G-CSF in this situation.

The different active substances (lenograstim, filgrastim, PEG-filgrastim) display equivalent effects [29] and can be used in the situations described above. GM-CSF (granulocyte-macrophage-colony stimulating factor) plays no role in febrile neutropenia.

\section{Prophylaxis with anti-infective agents}

There is good evidence of the efficacy of antibiotic anti-infective prophylaxis during neutropenia. We have been aware for many years that febrile episodes and documented infections can be reduced [32]. Fluorochinolones have been well studied. In a randomised trial, prophylaxis with levofloxacin was very effective in reducing episodes of fever, documented infections, especially gram-negative bacterial infections [33].
High-risk patients with profound neutropenia for more than 7 days also show a survival benefit. Despite the potential risk of inducing resistant pathogens [34, 35], antibiotic prophylaxis is recommended in this particular patient group. Patients after autologous stem-cell transplantation also benefit, but a survival advantage has not been observed. Therefore most guidelines do not recommend antibiotic prophylaxis in these patients.

Nor do low-risk patients with solid tumours or lymphoma benefit routinely from a prophylactic approach. Even if there is a reduction in febrile episodes, there is no difference in documented infections compared to the placebo group [36] in that patient population.

The duration of prophylaxis should start before neutropenia, that is after finishing chemotherapy and continued until the neutrophils rise above $500 / \mu$ or empirical antibiotic therapy is started.

Although the risk for fungal infection is relatively high after allogenic transplantation, we do not address this situation discussed in this paper.

Risk for candida infection is higher when the mucosal barrier has been damaged [37] e.g. induced by intensive chemotherapy. The risk for fungal infections is highest in patients with persistent, $[38,39]$ profound neutropenia. Patients undergoing intensive induction chemotherapy for acute leukaemia are at risk for candida and aspergillus infections [40]. Prophylactic approaches should cover both groups of fungal pathogens. A randomised study showed posaconazole to be superior to fluconazole or itraconazole [41]. Even if there are problems with absorption and drug interaction (coadministration with chemotherapy should be avoided), in the posaconazole group, the rate of aspergillus infections was reduced to $1 \%$, compared to $7 \%$ with the other antifungal agents. Posaconazole prophylaxis is therefore indicated in these patients.

In patients with prior invasive aspergillus infection who are expected to experience a chemotherapy-induced period of neutropenia especially of more than 7 days, a secondary prophylaxis with a mold-active antifungal agent is recommended.

\section{Conclusion}

Febrile neutropenia is one of the complications after chemotherapy carrying a relevant risk for death. It is now considered standard therapy in several high-risk patient groups to use anti-infective prophylaxis to prevent infection, and in patients with a risk for febrile neutropenia of $\geq 20 \%$, to use G-CSF. Besides the prophylactic approach, it is important to deal with febrile neutropenia by employing adequate diagnostic procedures, but not to delay the appropriate therapy. Empiric antibiotic treatment should start immediately. A change of antimicrobial therapy is mandatory after a sufficient duration of therapy and clinical deterioration or an unstable situation and might be considered in case of persisting fever. Here the coverage of the spectrum of pathogens by the anti-infective treatment has to be adapted to the risk profile of the patient. Knowledge of the local epidemic situation, especially for resistant pathogens is essential for an appropriate choice of calculated or empiric antibiotic therapy. Documented infections must be incorporated in the strategy, but the spectrum of anti-infective treatment should not be narrowed. 


\section{Conflict of interest}

Florian Weissinger received lecture honoraria from Chugai. Werner Heinz received research support from Astellas, Basilea, Gilead, MSD/Merck (formerly Essex/Schering-Plough) and Pfizer, is member of the scientifics advisory board of Merck and Pfizer and received lecture honoraria from Gilead, MSD/ Merck (formerly Essex/Schering-Plough) and Pfizer.

\section{References}

[1] Bodey GP, Buckley M, Sathe YS, Freireich EJ. Quantitative relationships between circulating leukocytes and infection in patients with acute leukemia. Annals of internal medicine, 64(2): 328-40, 1966.

[2] Winston DJ, Lazarus HM, Beveridge RA, et al. Randomized, doubleblind, multicenter trial comparing clinafloxacin with imipenem as empirical monotherapy for febrile granulocytopenic patients. Clin Infect Dis, 32(3): 381-90, 2001.

[3] Freifeld AG, Bow EJ, Sepkowitz KA, et al. Clinical practice guideline for the use of antimicrobial agents in neutropenic patients with cancer: 2010 update by the infectious diseases society of america. Clin Infect Dis, 52(4): e56-93, 2010.

[4] Klastersky J, Paesmans M, Georgala A, et al. Outpatient oral antibiotics for febrile neutropenic cancer patients using a score predictive for complications. J Clin Oncol, 24(25): 4129-34, 2006.

[5] Klastersky J, Paesmans M, Rubenstein EB, et al. The Multinational association for supportive care in cancer risk index: A multinational scoring system for identifying low-risk febrile neutropenic cancer patients. J Clin Oncol, 18(16): 3038-51, 2000.

[6] Klastersky J. Management of fever in neutropenic patients with different risks of complications. Clin Infect Dis, 39(Suppl. 1): S32-7, 2004.

[7] Klastersky J, Ameye L, Maertens J, et al. Bacteraemia in febrile neutropenic cancer patients. International journal of antimicrobial agents, 30(Suppl 1): S51-9, 2007.

[8] Zinner SH. Changing epidemiology of infections in patients with neutropenia and cancer: emphasis on gram-positive and resistant bacteria. Clin Infect Dis, 29(3): 490-4, 1999

[9] Cordonnier C, Buzyn A, Leverger G, et al. Epidemiology and risk factors for gram-positive coccal infections in neutropenia: toward a more targeted antibiotic strategy. Clin Infect Dis, 36(2): 149-58, 2003.

[10] Kumar A, Roberts D, Wood KE, et al. Duration of hypotension before initiation of effective antimicrobial therapy is the critical determinant of survival in human septic shock. Critical care medicine, 34(6): 1589-96, 2006.

[11] Bow EJ, Rotstein C, Noskin GA, et al. A randomized, open-label, multicenter comparative study of the efficacy and safety of piperacillintazobactam and cefepime for the empirical treatment of febrile neutropenic episodes in patients with hematologic malignancies. Clin Infect Dis, 43(4): 447-59, 2006.

[12] Cherif $\mathrm{H}$, Bjorkholm M, Engervall $\mathrm{P}$, et al. A prospective, randomized study comparing cefepime and imipenem-cilastatin in the empirical treatment of febrile neutropenia in patients treated for haematological malignancies. Scandinavian journal of infectious diseases, 36(8): 593-600, 2004

[13] Raad, II, Escalante C, Hachem RY, et al. Treatment of febrile neutropenic patients with cancer who require hospitalization: a prospective randomized study comparing imipenem and cefepime. Cancer, 98(5): 1039-47, 2003

[14] Wang FD, Liu CY, Hsu HC, et al. A comparative study of cefepime versus ceftazidime as empiric therapy of febrile episodes in neutropenic patients. Chemotherapy, 45(5): 370-9, 1999.

[15] Biron P, Fuhrmann C, Cure H, et al. Cefepime versus imipenemcilastatin as empirical monotherapy in 400 febrile patients with short duration neutropenia. CEMIC (Study Group of Infectious Diseases in Cancer). J Antimicrob Chemother, 42(4): 511-8, 1998.

[16] Feld R, DePauw B, Berman S, Keating A, Ho W. Meropenem versus ceftazidime in the treatment of cancer patients with febrile neutropenia: a randomized, double-blind trial. J Clin Oncol, 18(21):3690-8, 2000.

17] Vandercam B, Gerain J, Humblet Y, et al. Meropenem versus ceftazidime as empirical monotherapy for febrile neutropenic cancer patients. Ann Hematol, 79(3): 152-7, 2000

[18] Lindblad R, Rodjer S, Adriansson M, et al. Empiric monotherapy for febrile neutropenia--a randomized study comparing meropenem with ceftazidime. Scand J Infect Dis, 30(3): 237-43, 1998.

[19] Behre G, Link H, Maschmeyer G, et al. Meropenem monotherapy versus combination therapy with ceftazidime and amikacin for empirical treatment of febrile neutropenic patients. Ann Hematol, 76(2): 73-80, 1998.

[20] Bohme A, Shah PM, Stille W, Hoelzer D. Piperacillin/tazobactam versus cefepime as initial empirical antimicrobial therapy in febrile neutropenic patients: a prospective randomized pilot study. Eur J Med Res, 3(7): 324-30, 1998.

[21] Paterson DL, Ko WC, Von Gottberg A, et al. Outcome of cephalosporin treatment for serious infections due to apparently susceptible organisms producing extended-spectrum beta-lactamases: implications for the clinical microbiology laboratory. J Clin Microbiol, 39(6): 2206-12, 2001.

[22] Yahav D, Paul M, Fraser A, Sarid N, Leibovici L. Efficacy and safety of cefepime: a systematic review and meta-analysis. Lancet Infect Dis, 7(5): 338-48, 2007.

[23] Paul M, Soares-Weiser K, Grozinsky S, Leibovici L. Beta-lactam versus beta-lactam-aminoglycoside combination therapy in cancer patients with neutropaenia. Cochrane Database Syst Rev, (3): CD003038, 2003.

[24] Paul M, Borok S, Fraser A, Vidal L, Leibovici L. Empirical antibiotics against Gram-positive infections for febrile neutropenia: systematic review and meta-analysis of randomized controlled trials. The Journal of antimicrobial chemotherapy, 55(4): 436-44, 2005.

[25] Cometta A, Kern WV, De Bock R, et al. Vancomycin versus placebo for treating persistent fever in patients with neutropenic cancer receiving piperacillin-tazobactam monotherapy. Clin Infect Dis, 37(3): 382-9, 2003.

[26] Elting LS, Lu C, Escalante CP, et al. Outcomes and cost of outpatient or inpatient management of 712 patients with febrile neutropenia. J Clin Oncol, 26(4): 606-11, 2008.

[27] Maertens J, Theunissen K, Verhoef G, et al. Galactomannan and computed tomography-based preemptive antifungal therapy in neutropenic patients at high risk for invasive fungal infection: a prospective feasibility study. Clin Infect Dis, 41(9): 1242-50, 2005.

[28] Kern WV. Risk assessment and treatment of low-risk patients with febrile neutropenia. Clin Infect Dis, 42(4): 533-40, 2006.

[29] Kuderer NM, Dale DC, Crawford J, Lyman GH. Impact of primary prophylaxis with granulocyte colony-stimulating factor on febrile neutropenia and mortality in adult cancer patients receiving chemotherapy: a systematic review. J Clin Oncol, 25(21): 3158-67, 2007.

[30] Maher DW, Lieschke GJ, Green M, et al. Filgrastim in patients with chemotherapy-induced febrile neutropenia. A double-blind, placebo-controlled trial. Ann Intern Med, 121(7): 492-501, 1994.

[31] Garcia-Carbonero R, Mayordomo JI, Tornamira MV, et al. Granulocyte colony-stimulating factor in the treatment of high-risk febrile neutropenia: a multicenter randomized trial. J Natl Cancer Inst, 93(1): 31-8, 2001.

[32] Gafter-Gvili A, Fraser A, Paul M, Leibovici L. Meta-analysis: antibiotic prophylaxis reduces mortality in neutropenic patients. Ann Intern Med, 142(12 Pt 1): 979-95, 2005.

[33] Bucaneve G, Micozzi A, Menichetti F, et al. Levofloxacin to prevent bacterial infection in patients with cancer and neutropenia. N Engl J Med, 353(10): 977-87, 2005.

[34] Kern WV, Klose K, Jellen-Ritter AS, et al. Fluoroquinolone resistance of Escherichia coli at a cancer center: epidemiologic evolution and effects of discontinuing prophylactic fluoroquinolone use in neutropenic patients with leukemia. Eur J Clin Microbiol Infect Dis 24(2): 111-8, 2005

[35] Kaye KS, Kanafani ZA, Dodds AE, Engemann JJ, Weber SG, Carmeli Y. Differential effects of levofloxacin and ciprofloxacin on the risk for isolation of quinolone-resistant Pseudomonas aeruginosa. Antimicrob Agents Chemother, 50(6): 2192-6, 2006.

[36] Cullen M, Steven N, Billingham L, et al. Antibacterial prophylaxis after chemotherapy for solid tumors and lymphomas. N Engl J Med, 353(10): 988-98, 2005.

[37] Blijlevens NM, Donnelly JP, de Pauw BE. Impaired gut function as risk factor for invasive candidiasis in neutropenic patients. $\mathrm{Br} \mathrm{J}$ Haematol, 117(2): 259-64, 2002.

[38] Gerson SL, Talbot GH, Hurwitz S, Strom BL, Lusk EJ, Cassileth PA. Prolonged granulocytopenia: the major risk factor for invasive pulmonary aspergillosis in patients with acute leukemia. Ann Intern Med, 100(3): 345-51, 1984.

[39] Portugal RD, Garnica M, Nucci M. Index to predict invasive mold infection in high-risk neutropenic patients based on the area over the neutrophil curve. J Clin Oncol, 27(23): 3849-54, 2009.

[40] Pagano L, Caira M, Candoni A, et al. The epidemiology of fungal infections in patients with hematologic malignancies: the SEIFEM2004 study. Haematologica, 91(8): 1068-75, 2006.

[41] Cornely OA, Maertens J, Winston DJ, et al. Posaconazole vs. fluconazole or itraconazole prophylaxis in patients with neutropenia. $\mathrm{N}$ Engl J Med, 356(4): 348-59, 2007. 